\title{
The "Natural" History of Medically Treated Temporal Lobe Epilepsy: What Can an Evidence-Based Approach Tell Us?
}

\author{
Colin Bruce Josephson and Bernhard Pohlmann-Eden \\ Division of Neurology, Department of Medicine, Dalhousie University, Queen Elizabeth II Health Sciences Centre, \\ 1796 Summer Street, Halifax, Nova Scotia, Canada B3H 3 A7 \\ Correspondence should be addressed to Bernhard Pohlmann-Eden, b.pohlmann-eden@dal.ca
}

Received 14 September 2011; Accepted 5 December 2011

Academic Editor: Seyed M. Mirsattari

Copyright ( 92012 C. B. Josephson and B. Pohlmann-Eden. This is an open access article distributed under the Creative Commons Attribution License, which permits unrestricted use, distribution, and reproduction in any medium, provided the original work is properly cited.

\begin{abstract}
We systematically reviewed the literature to describe the "natural" history of medically treated temporal lobe epilepsy (TLE). No population-based studies recruiting incident cases of TLE irrespective of age exist. Prospective, population-based studies were limited to those recruiting only childhood-onset TLE or those reporting TLE as a subgroup of cohorts of focal epilepsies. Few studies have been performed in the "MRI era" limiting information on natural history secondary to specific pathologies. Available data suggests that TLE is highly variable, with unpredictable transient remissions and low rates of seizure freedom (30 to $50 \%)$. Etiology and failure of first and second drug seem to be the most important predictors for treatment prognosis. The role of initial precipitating injuries remains speculative, as imaging information of related events is either missing or conflicting. Prospective cohorts of new-onset TLE with long-term followup using advanced MRI techniques, timely EEG recordings, and assessments of psychiatric comorbidities are needed.
\end{abstract}

\section{Introduction}

Temporal lobe epilepsy (TLE) is the most frequent medically refractory epilepsy syndrome seen in epilepsy outpatient clinics. It has received considerable attention in recent years owing to the remarkable rates of remission that can be achieved through surgical intervention [1]. Mesial temporal lobe epilepsy (mTLE) associated with hippocampal sclerosis (mTLE-HS), a condition that can be detected by modern magnetic resonance imaging (MRI) techniques with a high sensitivity and specificity, is now the most common indication for epilepsy surgery.

To date, therapeutic advances in TLE have far outpaced our understanding of the natural history of the disorder. According to a recent International League Against Epilepsy (ILAE) commission report [2], the natural history of mTLEHS is characterized by key features such as a history of an initial precipitating injury and a presence of a latent and/or silent period. Prior publications have attempted to assess the course and prognosis of TLE, mTLE, and mTLEHS using sophisticated electroencephalography (EEG), MRI, and histological techniques trying to identify the "natural" history of all types of TLE. Almost all these studies are limited by the fact that their perspective comes from tertiary care centers and surgical series [3].

The ideal natural history study requires a large prospective cohort of patients with new-onset TLE undergoing extensive structural and functional testing with a followup of $>10$ years. We conducted a systematic review of the literature to identify prospective, population-based natural history studies of TLE and reviewed appropriate longitudinal and retrospective case series. We extracted critical information on initial precipitating injury, seizure outcome, silent periods, and long-term remission to describe the natural history of this condition. Natural history in its strictest sense means the course of a disorder from onset without intervention until the disorder resolves or death ensues [4]. However, data from drug-naïve TLE patients are only anecdotal. Therefore, for the purposes of this study, we defined the "natural history" of TLE as the course of the disorder under medical treatment without surgical intervention. 


\section{Methods}

We performed a comprehensive literature search of Medline and Embase using validated search terms (See the Appendix) on August 10th, 2011. We searched the bibliography of relevant reviews and articles to identify additional studies that may not have been retrieved by our search strategy. One of the authors (CBJ) independently applied the following study inclusion criteria: (i) unselected cohorts of epilepsy patients drawn from the general population; (ii) a minimal study population of $\geq 20$ participants; (iii) quantitative report of patient demographics, initial precipitating injuries, the results of EEG and MRI, and seizure outcomes. Titles were screened and abstracts or full articles were reviewed when there was doubt concerning the article's potential relevance. Questions concerning eligibility were resolved through consensus discussion between the two authors. All relevant articles were retrieved for full review. We abstracted data from the most recent publication if there were multiple articles identified from the same study population. Fulllength English articles were selected without restriction on place or date of publication.

We accepted the authors' diagnosis of TLE. One of the authors (CBJ) abstracted all data and priority was given to prospective, population-based cohorts of incident diagnoses of TLE. The primary outcome was seizure freedom at the end of followup. Age at onset, the frequency of risk factors such as a family history or initial precipitating injuries, frequency of abnormalities found on electrophysiological and neuroimaging studies, the existence of an intervening "silent" period between diagnosis and later seizure relapse, and the longest duration of remission were considered secondary outcomes of interest. Additional measures such as psychosocial and cognitive outcomes were not analyzed due to the limited scope of the paper.

\section{Results}

We identified 1774 articles and reviewed titles and abstracts to retrieve $39(2 \%)$ that were potentially relevant to the review. We performed a full review of all 39 articles and 5 $(13 \%)$ were ultimately included in our analysis (Figure 1). No population-based studies recruiting all incident cases of TLE irrespective of age exist. Prospective, population-based studies were limited to those recruiting only childhood-onset TLE or those reporting on TLE as a subgroup of populationbased cohorts of focal epilepsies (Table 1). Few studies have been performed in the "MRI era" thus limiting information on the natural history and prognosis of TLE secondary to specific underlying pathologies.

3.1. Seizure Outcome. Only one population-based study dedicated solely to TLE was identified by our search. It enrolled incident and prevalent cases of TLE in the state of Victoria, Australia, (interval from seizure onset to enrollment was 1 week to 3.9 years) and found that $31 \%$ of individuals were able to achieve a minimum of two-year seizure freedom off antiepileptic drugs (AEDs) over a median follow-up period of 13.7 years. All other individuals in the cohort either continued to have seizures despite AED status or had progressed to surgery. Interestingly, all patients still taking AEDs at the end of followup had failed to achieve seizure remission. However, only two children who went greater than one-year seizure free had a recurrence of seizures before their terminal remission. The only predictor of seizure freedom according to a univariable analysis was the absence of a lesion on MRI [5].

Additional prospective, population-based studies of focal epilepsies have been informative. The National General Practice Study of Epilepsy (NGPSE) captured incident diagnoses of epileptic seizures across the United Kingdom over a threeyear period from 1984-1987 [6]. They stratified patients into anatomical groups whereby $27 \%$ of the total population were classified as having seizures of a temporal lobe origin mainly based on clinical and EEG information. Patients were followed for 4 to 7 years and, of those with temporal lobe seizures, $49 \%$ achieved two-year seizure freedom while 33\% had less than four seizures per year. It is unclear whether any of the patients included in the follow-up analysis underwent surgical resection. A study of 1448 people with epileptic and febrile seizures from Rochester, MN, USA, found that $41 \%$ of individuals with temporal lobe epilepsy had achieved two-year seizure freedom at the point of last followup [7]. Likewise, a prospective population-based evaluation of 144 incident cases of childhood-onset generalized and focal epilepsy from Turku, Finland found that $53 \%$ of individuals with TLE were able to achieve seizure-freedom for five or more years at the end of a median of 40 years of followup (range 11 to 42). A further 19\% entered a five-year period of seizure remission at some point during the follow-up period [8].

There is little prospective information available for the prognosis of individual subtypes of TLE. A prospective study of incident and prevalent cases of familial mesial temporal lobe sclerosis suggested that its prognosis may not differ significantly from sporadic TLE. Patients were classified according to their degree of seizure freedom at the beginning of follow-up (seizure-free for two or more years, less than six complex partial seizures per year and no more than two secondarily generalized seizures per year, or six or greater complex partial seizures per year despite adequate AED therapy). At the end of a mean of 7.8 years followup (standard deviation of \pm 1.3 years), $50 \%$ of participants had achieved seizure freedom for two or more years. However, none of the patients who started the study with medically refractory epilepsy achieved seizure remission by the end of followup [9]. As expected, symptomatic family members appeared more likely to develop epilepsy (11\%) than those from the general population.

Retrospective studies from tertiary care centres reported far more variable rates of seizure remission. Rates of seizure freedom of one year or more by last followup ranged from $11-63 \%$ in populations of patients with TLE [10$16]$ or in cohort or subgroup analyses of those with mesial temporal lobe sclerosis $[10,17]$. When evaluated according to number of seizures per month, $37-61 \%$ had improved 


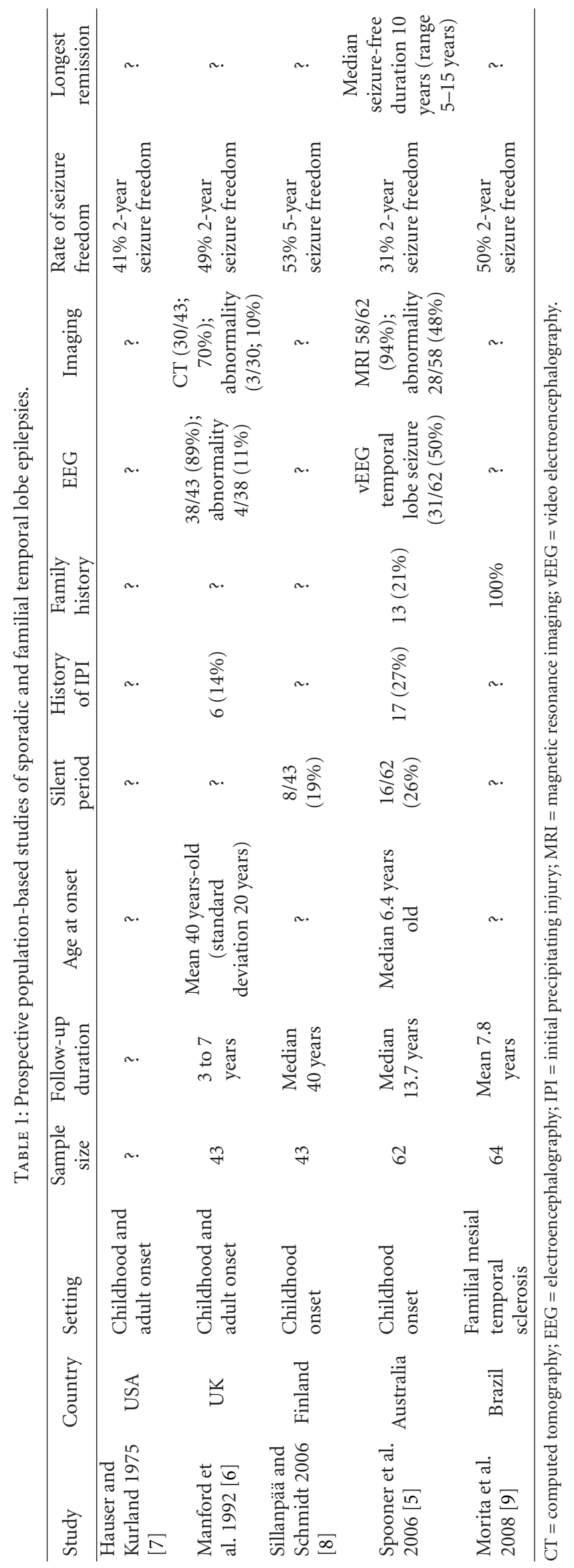




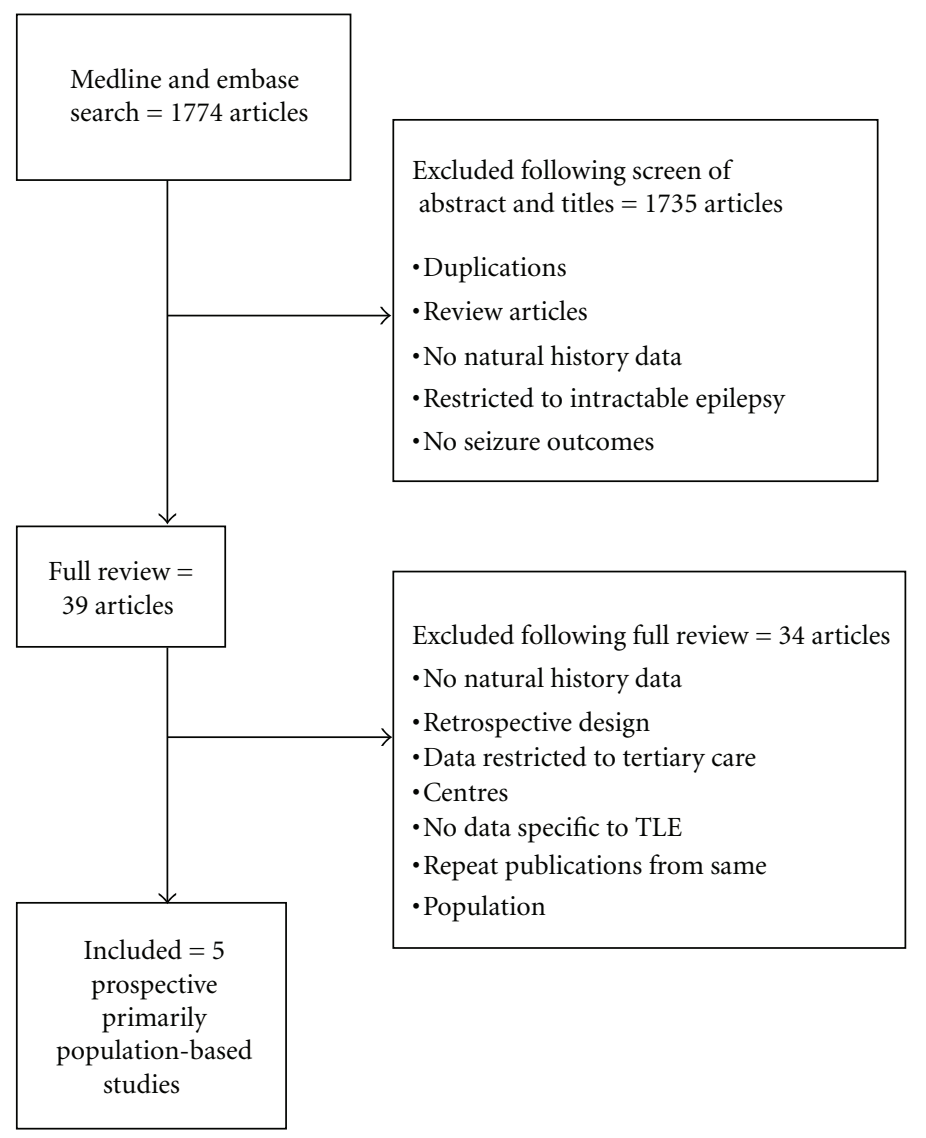

FIGURE 1: Search strategy and evidence base.

seizure control or $<1$ seizure per month after mean followups of up to 6.6 years. Those with idiopathic (normal neurological status and intelligence with no risk factors for epilepsy other than a family history of seizures) TLE showed an improved prognosis (79\% seizure free at last followup [minimum follow-up of two years] versus $52 \%$ in patients without idiopathic TLE) [13], as may those who respond successfully to their first AED (odds ratio for refractory TLE 93.8 [95\% confidence interval, 95\% CI, 20.1 to 437.8 ]) [16].

3.2. Initial Precipitating Injuries. Two studies reported on rates of potential precipitating injuries that may have contributed to the development of temporal lobe epilepsy. Common antecedent events considered risk factors for epilepsy included complicated febrile convulsions, prolonged afebrile seizures, infantile spasms, meningitis or encephalitis, hypertensive encephalopathy, apnea and respiratory arrest, tumours, congenital malformations, remote trauma, and vascular events including intracranial hemorrhage $[5,6]$. The reported frequency of events between cohorts differed considerably with an earlier study reporting an identified risk factor in 14\% of the population [6] compared to a later study in the MRI era that reported an identified risk factor in $27 \%$ of the cohort [5].
3.3. Abnormalities on Functional and Structural Tests. Two studies reported proportions of patients undergoing electrophysiological and neuroimaging studies. Electroencephalography (EEG) was performed in $89 \%$ of patients with temporal lobe epilepsy in one study and revealed evidence of a focal abnormality in $11 \%$ of those tested [6]. A second study reported evidence of seizures of temporal lobe origin on video-EEG telemetry in 50\% of the study cohort [5]. The percentage of patients undergoing neuroimaging varied between these two studies. Seventy percent of one cohort from the pre-MRI era underwent a CT scan of the head [6] while $94 \%$ of participants in a later study [5] underwent an MRI scan. A focal lesion was detected in $10 \%$ of those investigated with a CT scan of the brain (in which two of the three identified focal abnormalities, all of which were primary tumours, were located in an extratemporal area) [6] and in $48 \%$ of those undergoing MRI [5]. Temporal lobe lesions detected by MRI included hippocampal sclerosis in 10 children, tumour in 8 , cortical dysplasia in 7 , and temporal lobe atrophy, gliosis, or cystic change in 3 .

3.4. "Silent Periods". No studies reported the delay from the first seizure to the diagnosis of epilepsy. However, in one study of epilepsy in Rochester, MN, there were more cases of temporal lobe epilepsy in an undiagnosed subgroup whose 
epilepsy had developed but had not yet been localized to the temporal lobe on the dates from which cases were drawn to determine incidence and prevalence. A disproportionate number of these patients were eventually diagnosed with TLE suggesting a longer interval between initial seizure onset and ultimate epilepsy classification when compared to other types of epilepsy [7].

Few studies reported on periods of seizure remission followed by symptomatic relapse. One study of childhoodonset temporal lobe epilepsy, which included individual patient data, reported that 16 of 62 cases $(26 \%)$ had periods of at least one year of seizure freedom before relapsing; some of which occurred between five to ten years after the onset of epilepsy [5]. A similar finding was reported in a subgroup analysis of patients with temporal lobe epilepsy from another prospective, population-based study of new onset epilepsy. In this subgroup, $19 \%$ of patients with temporal lobe epilepsy failed to achieve complete seizure control following a period of at least 5 years of seizure freedom [8].

3.5. Longest Remission Period. Only one study reported on the duration of seizure freedom. Spooner et al. [5], in their study of childhood onset temporal lobe epilepsy from Victoria, Australia, reported a median duration of seizure freedom of 10 years in those who achieved complete remission. The range of seizure freedom extended from 5 to 15 years.

\section{Discussion}

This systematic review of the literature highlights the relative dearth of information available on the natural course of medically treated TLE. It supports the notion that our knowledge of the "natural" history of medically treated TLE is very poor. Clinical impression of the benign or malign nature of TLE typically derives from what is often a highly selected surgical series with potentially nonrepresentative patient populations. Patient groups in these studies mainly consist of medically refractory cases of TLE and are often restricted to the subpopulation of mTLE. This is further complicated by the fact that within the mTLE group, the focus has primarily been on mTLE-HS, which is only one well-described disorder in what is otherwise a wide range of heterogeneous conditions that cause mTLE [2]. A recent review of the natural history of mTLE [3] emphasized the importance of the age of onset (childhood versus adult form) and the relative complexity of its course. It may appear relatively benign at first, with high variable times of remission and seizure freedom, but then later develop into an absolutely medically refractory seizure disorder. The review describes a typical surgical TLE patient using a "3rule" pattern: the average patient is in his or her 3rd decade when diagnosed with drug resistance and identified as a surgical candidate, $1 / 3 \mathrm{rd}$ of all patients have a history of febrile seizures and two-thirds show hippocampal sclerosis on MRI [3].

Not surprisingly, our systematic review of the literature revealed many deficiencies in our knowledge and raised several questions. We did not identify any population-based studies recruiting all incident cases of temporal lobe epilepsy over the whole life span. Our extracted information was limited to few studies, including a prospective, populationbased study on childhood-onset temporal lobe epilepsy [5] or those where TLE was a subgroup of population-based cohorts of focal epilepsies [6-9]. Information on imaging protocols was poor and MRI data were often lacking.

Prognosis in general, measured by seizure freedom, was worst in the most rigorous prospective study of childhood onset-TLE. Only $31 \%$ of individuals were able to achieve a minimum of two-year seizure freedom off AEDs over a median follow-up period of 13.7 years [5], compared to $49 \%$ of TLE patients in a prospective, population-based study of focal epilepsies [6] and $41 \%$ of a subgroup of TLE patients in 1448 cases of epileptic and febrile seizures in the Rochester Study [7]. Familial "benign" mTLE had only a slighter better prognosis with $50 \%$ of participants achieving seizure freedom for $>2$ years [9]. All patients who failed initial AED therapy remained refractory to medical therapy throughout [9]. This finding is in keeping with a retrospective study in childhood temporal lobe epilepsy, in which failure of the first AED was the strongest predictor for outcome [16]. Both studies emphasize the predictive role of initial drug failure for long-term prognosis. Significant variations of rates of seizure freedom and very high rates of seizure freedom (in up to $63 \%$ ) were only found in the retrospective series [1016] underlining the importance of prospective studies. The high rate of seizure freedom in $79 \%$ of individuals with "idiopathic" TLE [13] has to be interpreted with caution due to the small retrospective nature of the series which could have lead to selection bias.

Initial precipitating injuries, including complicated febrile convulsions and nonspecific brain insults (meningitis or encephalitits), were found in $14 \%$ in the NGPSE study [6] and in almost double (27\%) the participants in the prospective childhood TLE study by Spooner et al. Most of these patients had complicated febrile convulsions, followed by meningitis and a diverse array of other conditions.

Sophisticated imaging is crucial to assess the potential causal role of associated temporal lobe pathology. While CT scans in the pre MRI-era NGPSE study only detected abnormalities in 10\% (mainly extratemporal tumours), almost half of all patients (48\%) in the Australian study [5] had subtle lesions on MRI with an equal distribution of hippocampal sclerosis, cortical dysplasia, and tumours. The presence of a temporal lesion was the only significant predictor for long-term refractoriness to medical therapy. While spontaneous remission in this childhood cohort occurred in one-third of all patients over a median followup of 13.7 years, the remaining two-thirds with intractable seizures all had a lesion on MRI at seizure onset. The issue of hippocampal pathology on MRI (signal increase and atrophy) in newly diagnosed epilepsy and new-onset epilepsy remains controversial as it may precede seizure occurrence [18], it may be entirely missed or absent at onset [19-21], or hippocampal atrophy and volume loss can develop over the next three to four years $[19,22]$. Furthermore, nearly $40 \%$ of cases of "benign" mTLE (in which patients remain 
seizure free for at least 24 months with or without AEDs) displays evidence of hippocampal sclerosis on MRI [23]. These conflicting data reflect the ongoing debate of whether mesial temporal lobe atrophy in patients with TLE is a cause or consequence of repeated seizures [24]. Our search only retrieved two studies that documented EEG results $[5,6]$, and these surprisingly added only limited information to the natural course of TLE. The NGPSE study found focal abnormalities in one of every ten TLE patients and did not comment on their prognostic value. In the Australian study, focal EEG abnormalities were associated with poor seizure outcome $(P<0.05)$, but these results were strongly interrelated with corresponding MRI findings that were highly predictive of seizure intractability $(P<0.001)$.

Based on this systematic review of the literature, we currently cannot predict whether the course of new-onset TLE will be straightforward with rapid long-term seizure control, rapidly progress to drug resistance, or go into shortor long-term remission. Stuttering, relapsing, and silent periods may be a characteristic features of TLE. Over onequarter $(26 \%)$ of the prospective childhood-onset temporal lobe epilepsy had periods of at least one year of seizure freedom before relapsing [5]. Almost every fifth patient in a prospective, population-based study with a seizure-free period of 5 years turned out to be refractory over the long term [8]. This is in keeping with another prospective study of children with focal seizures (not TLE only) where $76 \%$ of those patients who became finally refractory to medical therapy had a preceding remission of at least one year. Interestingly, however, failing two or more AEDs did not preclude remission times of greater 1 year [25]. Current data do not offer much useful information on whether the duration of seizure freedom can be used to predict overall long-term seizure remission. Only one study has directly addressed this issue reporting on childhood onset TLE from Victoria, Australia, where seizure freedom ranged from 5 to 15 years for those who finally became seizure free [5].

4.1. Where Does This Information Take Us? Our systematic review confirmed that the course TLE could be highly variable with unpredictable phases of transient remissions and low rates of seizure freedom that range between 30 and $50 \%$ in prospective studies. Based on the limited available data, etiology and failure of first and second AED are the most important predictors for prognosis. The role of initial precipitating injuries remains speculative, as imaging information of related events is either missing or conflicting. Our understanding of the basic mechanisms of epileptogenesis remains limited.

Prospective, population based studies of new-onset epilepsies suggestive of TLE are needed to adequately address these questions in the context of a reliable, evidencebased framework. For instance, a rigorous approach to determining epilepsy syndromes upon evaluating the first seizure (including new-onset epilepsy) was adopted by King et al. (1998) [26] using MRI in up to $90 \%$ of their patients and early EEG (within 24 hours of the seizure) plus sleepdeprived EEG, if routine EEG was normal. The authors were able to classify localization-related or generalized syndromes in only $47 \%$ of the cases with clinical information versus $77 \%$ of those with the addition of EEG findings and $81 \%$ of those with the addition of MRI. Their findings emphasize the clinical usefulness of EEG, which was found to be superior to MRI in classifying epilepsy syndromes, although the low yield may have been improved by the use of modern MRI techniques that were not available during the study period of 1994-1996. Our systematic review highlights the paucity of evidence-based longitudinal data on the use of EEG over the course of TLE. This is an important observation since EEG findings are the most relevant predictor for seizure recurrence besides etiology and first-seizure presentation [27].

There is now considerable interest in the role of advanced neuroimaging technology in early stages of epilepsy [28, 29]. The First Halifax International Epilepsy Conference in September, 2010, which focused on the role of advanced neuroimaging in new-onset epilepsy, reached an expert consensus that first seizures and new-onset epilepsy most likely reflect a change in the complex biochemical and neurobiologic environment of the brain in which seizures themselves only represent one key symptomatic feature [29]. It therefore seems logical to apply the same sophisticated structural and functional investigations that have been developed for study of chronic stages of epilepsy to the first seizure and newly diagnosed epilepsy. A careful evaluation of preceding psychiatric comorbidities, such as anxiety and depression, is also required as they often reflect temporal pathology as part of a bidirectional relationship between epilepsy and psychiatric findings patients with TLE [29, 30]. It is likely that advanced MRI techniques including diffusion tensor imaging and MR spectroscopy, in addition to timely EEG recordings, will provide new insights into the early course of TLE and help to identify predictors for pharmacoresistance and further define the potential dynamic course of TLE [28]. Extensive ongoing followup of these prospective cohorts of new-onset epilepsy or newly diagnosed TLE is needed as highly variable, and unpredictable phases of intermittent remission can occur over the long-term "natural" course of medically treated TLE.

\section{Appendix}

\section{Medline and Embase Search Strategy}

(1) exp epilepsy, temporal lobe/

(2) ((epilepsy adj3 temporal) or TLE).tw.

(3) exp incidence/

(4) exp mortality/

(5) exp prognosis/

(6) exp quality of life/

(7) exp survival analysis/

(8) (incidence or mortality or prognos* or predict* or course or outcome or "quality of life" or memory or "survival analysis").tw. 
(9) $\exp$ cohort study/

(10) exp case-control study/

(11) exp Randomized Controlled Trial/

(12) ("randomi* control* trial" or cohort or risk or casecontrol).tw.

(13) exp followup studies/

(14) ( 1 or 2$)$ and ( 3 or 4 or 5 or 6 or 7 or 8 ) and ( 9 or 10 or 11 or 12 or 13 )

(15) limit 14 to humans.

\section{Disclosures}

Dr. C. Josephson reports no disclosure. Dr. B. PohlmannEden reports no disclosure.

\section{References}

[1] S. Wiebe, W. T. Blume, J. P. Girvin, and M. Eliasziw, "A randomized, controlled trial of surgery for temporal-lobe epilepsy," New England Journal of Medicine, vol. 345, no. 5, pp. 311-318, 2001.

[2] H.-G. Wieser, "ILAE Commission on Neurosurgery of Epilepsy: ILAE Commission Report. Mesial temporal lobe epilepsy with hippocampal sclerosis," Epilepsia, vol. 45, no. 6, pp. 695-714, 2004.

[3] A. T. Berg, "The natural history of mesial temporal lobe epilepsy," Current Opinion in Neurology, vol. 21, no. 2, pp. 173 178, 2008.

[4] J. Last, A Dictionary of Epidemiology, Oxford Press, New York, NY, USA, 1983.

[5] C. G. Spooner, S. F. Berkovic, L. A. Mitchell, J. A. Wrennall, and A. S. Harvey, "New-onset temporal lobe epilepsy in children: lesion on MRI predicts poor seizure outcome," Neurology, vol. 67, no. 12, pp. 2147-2153, 2006.

[6] M. Manford, Y. M. Hart, J. W. A. S. Sander, and S. D. Shorvon, "National General Practice Study of Epilepsy (NGPSE): partial seizure patterns in a general population," Neurology, vol. 42, no. 10, pp. 1911-1917, 1992.

[7] W. A. Hauser and L. T. Kurland, "The epidemiology of epilepsy in Rochester, Minnesota, 1935 through 1967," Epilepsia, vol. 16, no. 1, pp. 1-66, 1975.

[8] M. Sillanpää and D. Schmidt, "Natural history of treated childhood-onset epilepsy: prospective, long-term populationbased study," Brain, vol. 129, no. 3, pp. 617-624, 2006.

[9] M. E. Morita, L. Conz, C. V. Maurer-Morelli et al., "Long term follow up of familial mesial temporal lobe epilepsy," Journal of Epilepsy and Clinical Neurophysiology, vol. 14, no. 3, pp. 111113, 2008.

[10] K. Aso and K. Watanabe, "Limitations in the medical treatment of cryptogenic or symptomatic localization-related epilepsies of childhood onset," Epilepsia, vol. 41, supplement 9, pp. 18-20, 2000.

[11] B. M. Soeder, U. Gleissner, H. Urbach et al., "Causes, presentation and outcome of lesional adult onset mediotemporal lobe epilepsy," Journal of Neurology, Neurosurgery and Psychiatry, vol. 80, no. 8, pp. 894-899, 2009.
[12] M. Harbord and J. Manson, "Temporal lobe epilepsy in childhood: reappraisal of etiology and outcome," Pediatric Neurology, vol. 3, no. 5, pp. 263-268, 1987.

[13] G. Regesta and P. Tanganelli, "Temporal lobe epilepsy of adult age of possible idiopathic nature," Seizure, vol. 11, no. 2, pp. 131-135, 2002.

[14] D. Chao, J. A. Sexton, and L. S. Santos Pardo, “Temporal lobe epilepsy in children," The Journal of Pediatrics, vol. 60, no. 5, pp. 686-693, 1962.

[15] S. Currie, K. W. G. Heathfield, R. A. Henson, and D. F. Scott, "Clinical course and prognosis of temporal lobe epilepsy: a survey of 666 patients," Brain, vol. 94, no. 1, pp. 173-190, 1971.

[16] D. J. Dlugos, M. D. Sammel, B. L. Strom, and J. T. Farrar, "Response to first drug trial predicts outcome in childhood temporal lobe epilepsy," Neurology, vol. 57, no. 12, pp. 22592264, 2001.

[17] W. J. Kim, S. C. Park, S. J. Lee et al., "The prognosis for control of seizures with medications in patients with MRI evidence for mesial temporal sclerosis," Epilepsia, vol. 40, no. 3, pp. 290293, 1999.

[18] K. E. VanLandingham, E. R. Heinz, J. E. Cavazos, and D. V. Lewis, "Magnetic resonance imaging evidence of hippocampal injury after prolonged focal febrile convulsions," Annals of Neurology, vol. 43, no. 4, pp. 413-426, 1998.

[19] T. Salmenperä, M. Könönen, N. Roberts, R. Vanninen, A. Pitkänen, and R. Kälviäinen, "Hippocampal damage in newly diagnosed focal epilepsy: a prospective MRI study," Neurology, vol. 64 , no. 1, pp. 62-68, 2005.

[20] R. Kälviäinen, T. Salmenperä, K. Partanen, P. Vainio, P. Riekkinen, and A. Pitkänen, "Recurrent seizures may cause hippocampal damage in temporal lobe epilepsy," Neurology, vol. 50, no. 5, pp. 1377-1882, 1998.

[21] W. Van Paesschen, J. S. Duncan, J. M. Stevens, and A. Connelly, "Longitudinal quantitative hippocampal magnetic resonance imaging study of adults with newly diagnosed partial seizures: one-year follow-up results," Epilepsia, vol. 39, no. 6, pp. 633639, 1998.

[22] R. S. N. Liu, L. Lemieux, J. W. A. S. Sander et al., "Seizureassociated hippocampal volume loss: a longitudinal magnetic resonance study of temporal lobe epilepsy," Annals of Neurology, vol. 51, no. 5, pp. 641-644, 2002.

[23] A. Labate, A. Gambardella, E. Andermann et al., "Benign mesial temporal lobe epilepsy," Nature Reviews Neurology, vol. 7, no. 4, pp. 237-240, 2011.

[24] F. Cendes, F. Andermann, P. Gloor et al., "Atrophy of mesial structures in patients with temporal lobe epilepsy: cause or consequence of repeated seizures?" Annals of Neurology, vol. 34, no. 6, pp. 795-801, 1993.

[25] A. T. Berg, B. G. Vickrey, F. M. Testa et al., "How long does it take for epilepsy to become intractable? A prospective investigation," Annals of Neurology, vol. 60, no. 1, pp. 73-79, 2006.

[26] M. A. King, M. R. Newton, G. D. Jackson et al., "Epileptology of the first-seizure presentation: a clinical, electroencephalographic, and magnetic resonance imaging study of 300 consecutive patients," The Lancet, vol. 352, no. 9133, pp. 10071011, 1998.

[27] B. Pohlmann-Eden and M. Newton, "First seizure: EEG and neuroimaging following an epileptic seizure," Epilepsia, vol. 49, supplement 1, pp. 19-25, 2008.

[28] M. H. Schmidt and B. Pohlmann-Eden, "Neuroimaging in epilepsy: the state of the art," Epilepsia, vol. 52, supplement 4, pp. 49-51, 2011. 
[29] B. Pohlmann-Eden, "Conceptual relevance of new-onset epilepsy," Epilepsia, vol. 52, supplement 4, pp. 1-6, 2011.

[30] A. M. Kanner, "Depression and epilepsy: a bidirectional relation?” Epilepsia, vol. 52, supplement 1, pp. 21-27, 2011. 


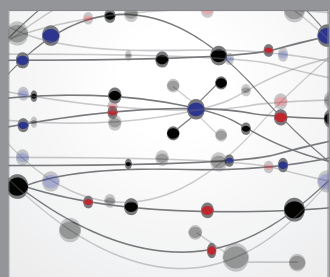

The Scientific World Journal
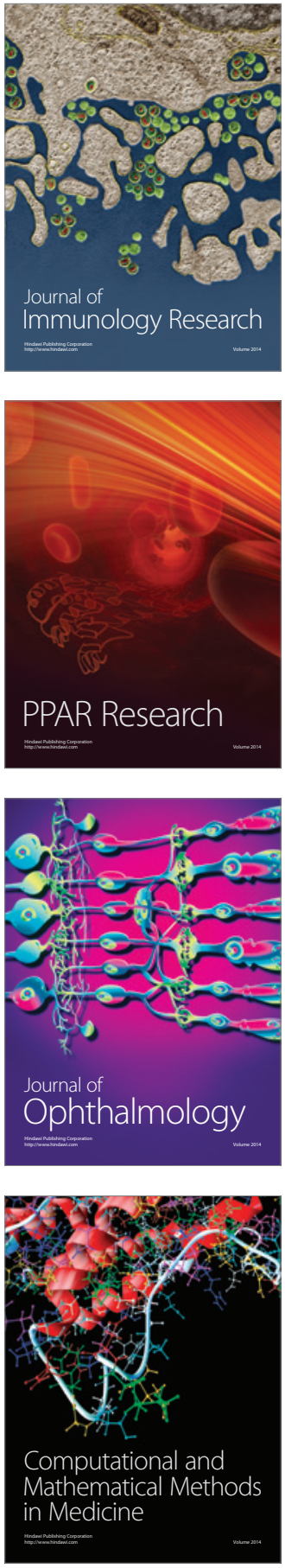

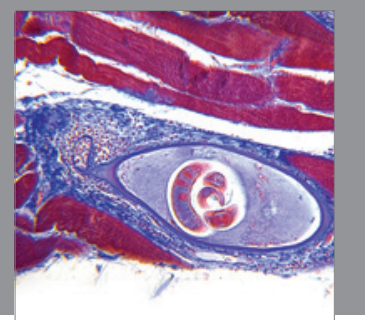

Gastroenterology

Research and Practice
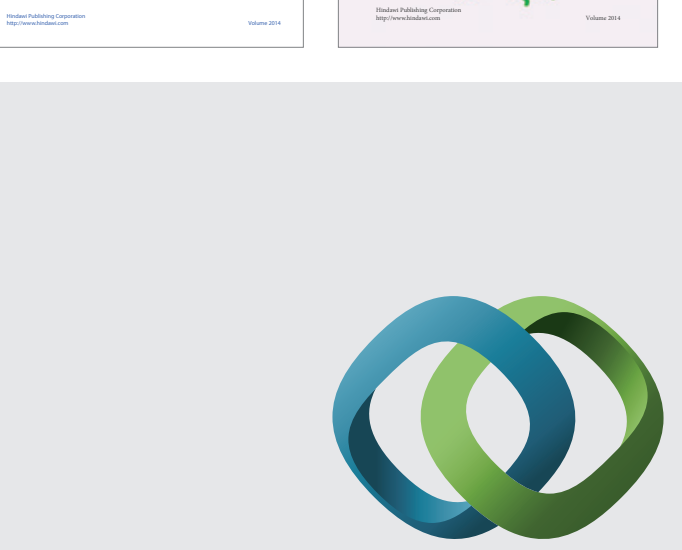

\section{Hindawi}

Submit your manuscripts at

http://www.hindawi.com
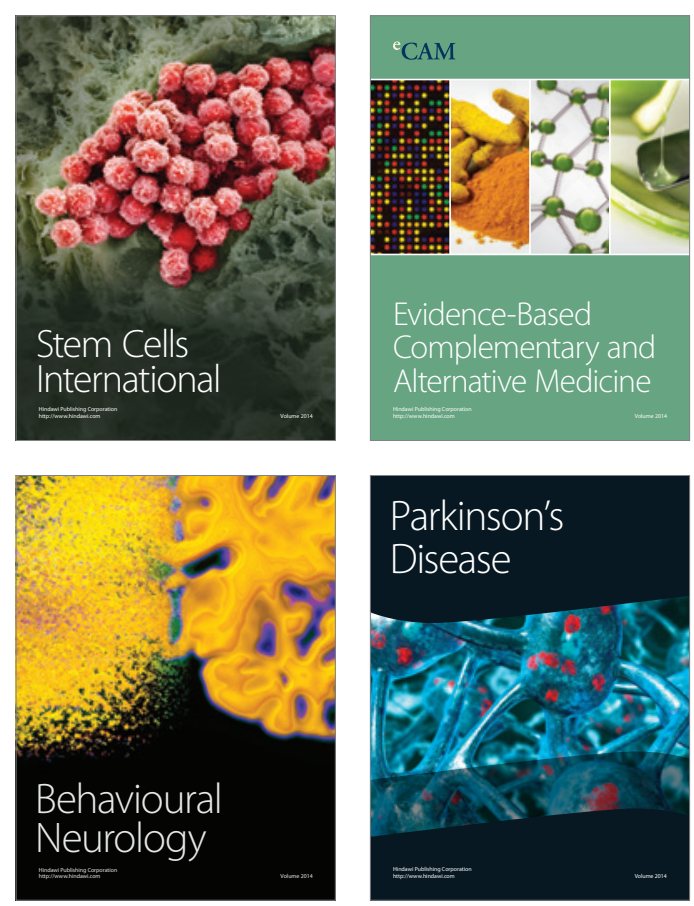

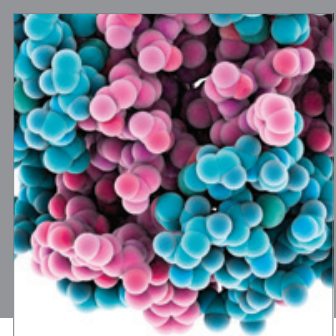

Journal of
Diabetes Research

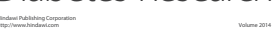

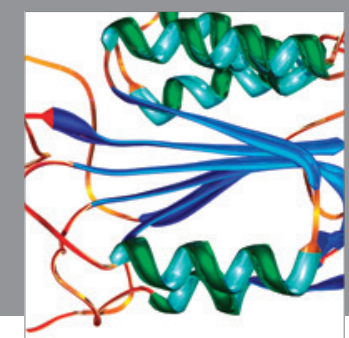

Disease Markers
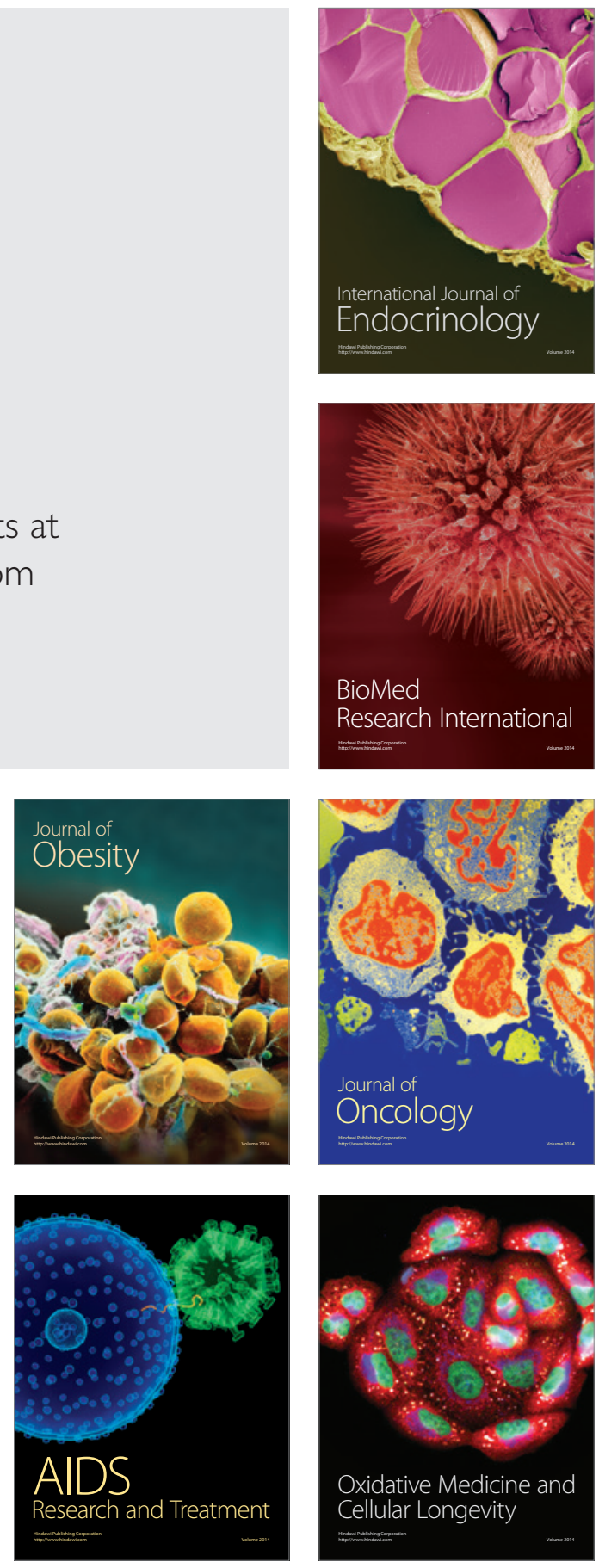